\title{
GENERALIZED FOURIER SERIES - A USEFUL MATHEMATICAL TOOL IN POWER ELECTRONICS
}

\author{
A. Jan Iwaszkiewicz ${ }^{1}$, B. Jacek Perz ${ }^{1}$, C. Manuel Perez Donsión ${ }^{2}$ \\ 1. The Electrotechnical Institute, Gdansk Branch, Poland \\ 2. Electrical Engineering Department, Vigo University, Spain
}

\begin{abstract}
The paper is related to the problem of creating stepped waveforms by use the generalized Fourier series. This method can be applied especially in power electronics as a tool permitting to reach AC voltage waveforms approximated to the sine wave shape. This novel approach to the synthesis of AC voltage waveforms is based on Fourier series of orthogonal functions set. Applying analytical methods and expressions given in the paper it is possible to define such optimal parameters of the waveform in order to receive the lowest harmonics content. The presented mathematical tool is very useful in designing structures and control algorithms of multilevel converters.
\end{abstract}

Key words - Fourier series, orthogonal functions, approximation, harmonics, multilevel converters

\section{INTRODUCTION}

In power electronics there are many devices i.e. $\mathrm{AC}$ drives, uninterruptible power sources or components of distributed power generation systems, where the essential demand is to generate sine wave voltage (or current) waveforms of relatively high quality. The voltage or current obtained from DC sources like rectifiers, storage accumulators, wind or photovoltaic farms and fuel cells is converted to AC using power electronics converters. The result of conversion may assume a shape of rectangular pulses or stepped waveforms. Their quality should comply with the appropriate standards. In mathematics the quality of approximation is measured by use of the average square error but in power electronics applications the most popular and useful tool is the THD factor (Total Harmonic Distortion). Although this factor does not carry any handy information about the shape of the harmonics spectrum.

The commonly used device for the purpose of energy conversion is two level VSI (Voltage Source Inverter), controlled using PWM (Pulse Width Modulation) method. But in VSIs the admissible method of conversion has well known disadvantages, related to high frequency switching, like power losses in switching elements and necessity of using special filters for high frequency components in the output voltages. These disadvantages can be reduced using multilevel converters and amplitude modulation method.

The paper presents a mathematical approach to the control strategy of multilevel converters. The relevant method for synthesis of the output waveforms applying generalised Fourier series is discussed [1,2]. Thanks to obtained useful and analytic description it is easy to optimise the parameters of the converter output waveforms. The mathematical waveform synthesis presented in the paper is a very helpful tool during designing of control algorithms and structures of multilevel inverters.

\section{WAVEFORMS SYNTHESIS BASED ON FOURIER SERIES}

\subsection{STANDARD FOURIER APPROXIMATION}

Given is a scaling function $\varphi(x)$ :

$$
\varphi(x)=\left\{\begin{array}{ll}
1 & \text { for } 0 \leq x<\alpha, \\
0 & \text { for other } x
\end{array}\right\} \quad \alpha \neq 0
$$

The scaling function $\varphi_{n}(x)$ is defined as follows:

$$
\varphi_{n}(x)=\varphi(x-n \alpha) \text { for } n=. .,-2,-1,0,1,2, . .
$$

The equation defines a set of rectangular pulses of unitary amplitude and an angle duration equal to $\alpha$. The pulse position on $x$ axis depends on parameter $n$ so setting this parameter permits to control the time delay of a pulse. A few examples of the scaling functions are presented in Fig. 1.

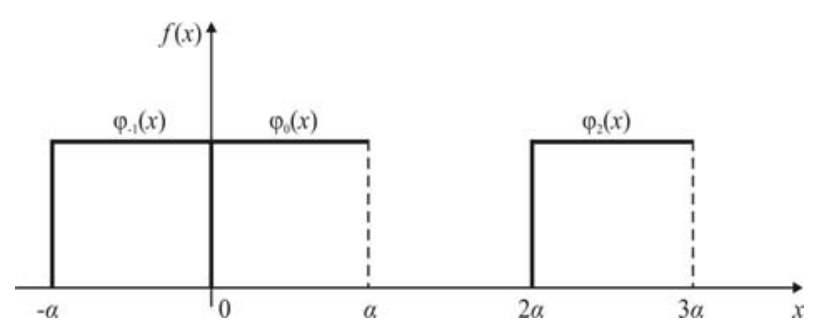

Fig. 1. Examples of the scaling functions: $\varphi_{-1}(x), \varphi_{0}(x), \varphi_{2}(x)$ 
In an interval $x \in<a, b)$, which length is $k \alpha(k \geq 1)$, functions $\varphi_{n}(x)$ satisfy two conditions:

$$
\begin{gathered}
\left\|\varphi_{n}\right\|^{2}=\int_{a}^{b} \varphi_{n}{ }^{2}(x) d x=\alpha \\
\int_{a}^{b} \varphi_{k}(x) \varphi_{m}(x) d x=0 \quad \text { if } \quad k \neq m
\end{gathered}
$$

therefore the notation (2) creates the set of orthogonal functions called an orthogonal base. Since all functions $\varphi_{n}(x)$ have their norms $\left\|\varphi_{n}\right\|^{2}$ equal to $\alpha$, this base is called an orthonormal base.

The expansion of a function $f(x)$ in a generalized Fourier series related to the set of scaling functions $\left(\varphi_{n}\right)$ is as follows:

$$
f(x)=\sum_{n=0}^{\infty} c_{n} \varphi_{n}(x)
$$

where $\quad c_{n}=\frac{\left(f, \varphi_{n}\right)}{\|\varphi\|^{2}}=\frac{\int_{a}^{b} f(x) \varphi_{n}(x) d x}{\alpha}$

The expansion (4) is valid for any function $f(x) \in L_{<a, b>}^{2}$ The Fourier series contains an infinite number of elements and makes it possible to approximate a function $f(x)$ by use of an infinite set (a sum) of adequately scaled functions $\varphi_{n}(x)$. Particularly it is possible to expand a function $f(x)=\sin (x)$ by summing an infinite set of rectangular pulses. It is in contradistinction to typical application of the Fourier series where any function $f(x)$ is expanded as a set of harmonics. According to (4) and (5) the expansion of $\sin (x)$ in the interval $x \in<a, b)$ is given as:

$$
\sin (x)=\sum_{n=0}^{n=\infty}\left\{\frac{\int_{a}^{b} \sin (x) \varphi_{n}(x) d x}{\alpha} \varphi_{n}(x)\right\} \quad x \in<a, b>
$$

The expression (6) defines a series of consecutive rectangular pulses represented by functions $\varphi_{n}(x)$. Amplitudes of pulses are different and determined after calculation of the integral. This statement can be applied for composition of sine waveforms in power electronics. Rectangular pulses represent an essential shape of output voltage (current) of an inverter. Naturally the composition of stepped waveforms using rectangular pulses has been applied in many applications but it concerned a "vertical" addition of waveforms. The resulting phase voltage was synthesized by the addition of the voltages generated by different cells of a cascaded inverter. The presented proposal relates to the addition of pulses "along $x$ axis" or in a time scale. Consecutive pulses form the resulting output voltage or current of the converter.

Practically in power electronics applications, the approximation of a sine wave can be realized using a finite number $N$ of the series members and natural aspiration of designers is to utilize the possibly lowest $N$ number. The accuracy of approximation depends on it. Its numerical value can be measured in different ways. In mathematics the accuracy of approximation is defined as the average square error $\delta$, a very useful criterion destined to that purpose. If the approximation of the function $f(x)=\sin (x)$ has been done in the interval $<a, b>$ by use of $N$ elements, the average square error $\delta$ is determined by the following expression:

$$
\begin{gathered}
\delta=\frac{1}{b-a} \int_{a}^{b}\left[\sin (x)-\sum_{n=0}^{N-1} c_{n} \varphi_{n}(x)\right]^{2} d x \\
x \in<a, b>, \quad b>a
\end{gathered}
$$

In power electronics the most important criterion of the accuracy or rather the quality of approximated waveforms is THD factor. For illustration of the problem two examples of approximation have been presented in Fig. 2 and Fig. 3. The harmonic spectra have been located near the waveforms in order to expose the evident relation between the number of steps and the order of appearing harmonics. Figures 2 and 3 present stepped waveforms obtained after approximation based on the set defined according to (1) and (2).

The results of Fourier approximation for selected $N$ are collected in Table 1. The results of average square errors, calculated according to the expression (7), are presented in the column denoted by $\delta_{N}$. The symbol $F_{N}$ denotes the number of steps in the scanned interval herein it is the interval $\langle 0,2 \pi\rangle$. The parameter $N_{\left|F_{N}\right|}$ denotes the number of demanded supply DC voltage or current sources. The correlation between these two parameters is described by the following: statement:

$$
\begin{gathered}
\text { if }\left(\frac{F_{N}}{4}\right) \in W \text { than } N_{\left|F_{N}\right|}=\left(\frac{F_{N}}{4}\right) \\
\text { else } N_{\left|F_{N}\right|}=E\left\{\frac{F_{N}}{4}+1\right\}
\end{gathered}
$$

where $W$ denotes the set of whole numbers and $E-$ function $[x]$ known as function Entier $\{x\}$. The value $N_{\left|F_{N}\right|}$ is a very important parameter of multilevel converters.

Table 1: The parameters of Fourier approximation for different $N$

\begin{tabular}{|c|c|c|c|c|}
\hline$F_{N}$ & $\alpha$ & $N_{\left|F_{N}\right|}$ & $\delta_{N}$ & THD \\
\hline$F_{N=2}$ & $\pi$ & 1 & 0.0947 & $48.37 \%$ \\
\hline$F_{N=6}$ & $\pi / 3$ & 2 & 0.0440 & $31.09 \%$ \\
\hline$F_{N=12}$ & $\pi / 6$ & 3 & 0.0113 & $15.23 \%$ \\
\hline$F_{N=16}$ & $\pi / 8$ & 4 & 0.0064 & $11.41 \%$ \\
\hline$F_{N=24}$ & $\pi / 12$ & 6 & 0.0028 & $7.63 \%$ \\
\hline
\end{tabular}

Fig. 2 presents the stepped waveform obtained using a very low approximation level. The ratio of the 
measures of steps is equal to 2 and it is the same as in a three phase inverter with connected load and controlled by an adequate set of rectangular waves. In order to produce such a waveform in one phase applications the converter needs only two DC sources easy to get e. g. by dividing one DC supply voltage.

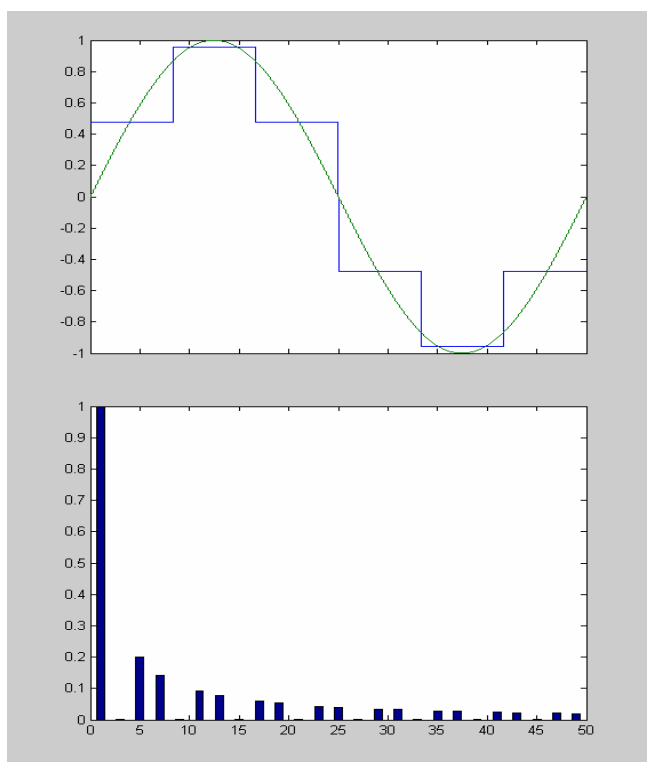

Fig. 2. The approximation of the function $f(x)=\sin (x)$ for $N=6$ $(\alpha=\pi / 3)$ and the spectrum analysis of the waveform

\subsection{OPTIMIZED FOURIER APPROXIMATION}

The proportion of step measures presented in Fig. 2 does not assure the minimal harmonic content of the waveform available in a converter equipped with only two DC sources. It will be shown further that changing the ratio of the supply voltages and matching the duration of pulses it is possible to decrease the THD factor of the output waveform.

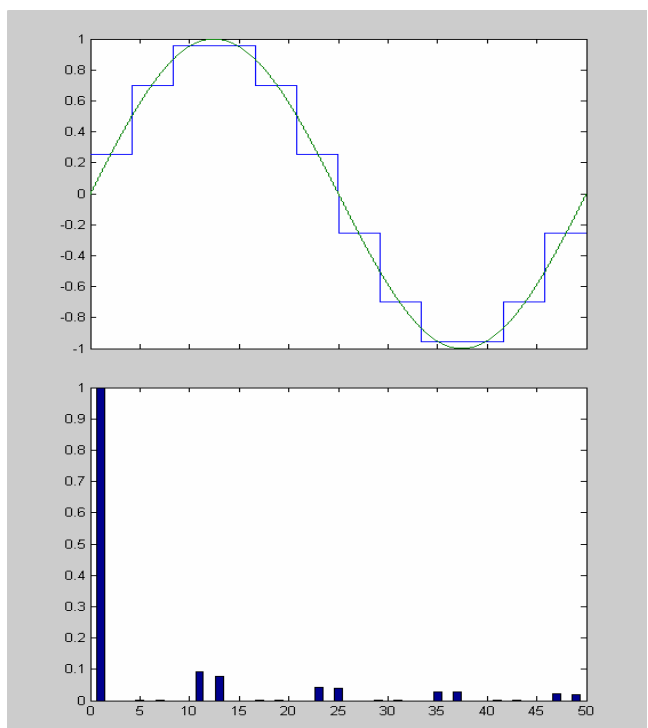

Fig. 3. The approximation of the function $f(x)=\sin (x)$ for $N=12(\alpha=\pi / 6)$ and the spectrum analysis of the waveform

Generally the application of the Fourier series in the domain of approximation does not demand the use of an orthonormal base of scaling functions. The necessary condition is the orthogonality of the base. Therefore another set of orthogonal functions can be applied for approximation. It may be a set consisting of rectangular pulses of different length $\alpha_{k}$ on the $x$ axis:

$$
\varphi_{k}(x)=\left\{\begin{array}{ccc}
1 & \text { for } & 0 \leq x<\alpha_{k}, \\
0 & \text { for } \text { other } x
\end{array}\right\} \quad \alpha_{k}>0, \quad k=0,1,2, \ldots
$$

The scaling function $\varphi_{n}(x)$ is defined as follows:

$$
\varphi_{n}(x)=\varphi\left(x-\sum_{k=0}^{k=n-1} \alpha_{k}\right) \text { for } \quad n=0,1,2, \ldots
$$

An example of approximation using two scaling functions: $\varphi_{0}(x) \varphi_{1}(x)$ has been presented in Fig. 4. Relevant parameters are: $\alpha_{0}=\pi / 6$ and $\alpha_{1}=2 \pi / 3$. In this case the harmonic spectrum is not regular and contains lower order harmonics including the third one. The total harmonic content is less than in case presented in Fig. 2: $\mathrm{THD}=21.62 \%$ in comparison to $31.09 \%$ in case of standard approximation. The approximation accuracy reaches the value $\delta=0.0222$.

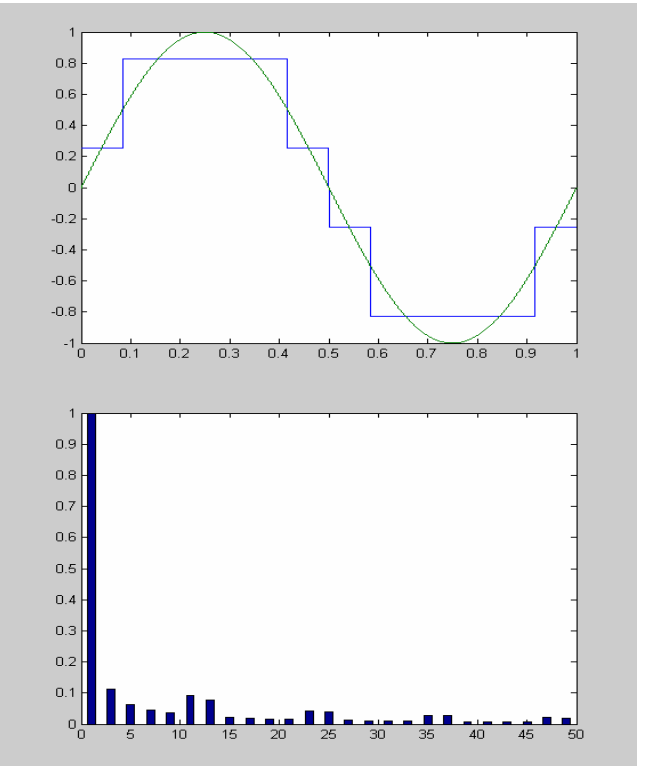

Fig. 4. The approximation of the function $f(x)=\sin (x)$ for $N=2$ $\left(\alpha_{0}=\pi / 6, \alpha_{1}=2 \pi / 3\right)$ and the spectrum analysis of the waveform

In case of the waveform presented in Fig. 2 all even harmonic components are equal to zero and odd components $b_{k}$ are given as:

$$
b_{k}=\frac{4}{k \pi}\left[V_{1}+\left(V_{2}-V_{1}\right) \cos \alpha\right] \quad k \in \mathrm{N}
$$


where $V_{1}$ and $V_{2}$ denote the measures of steps and $\boldsymbol{\alpha}$ the angle of the first step. It was assumed here that in every half cycle the waveform was symmetrical with respect to the straight line $\alpha=\frac{\pi}{2}+n \pi \quad n=. .,-2,-1,0,1,2, \ldots$. The relation-ship (11) can be utilized to select the step levels in order to cancel selected harmonic components. The problem of the selective harmonic elimination is well known as a fundamental of the state of the art. The example discussed here shows only the results available in the three level converter. Assuming canceling of third and fifth harmonic components a set of equation can be written:

$$
\left\{\begin{array}{l}
4\left[V_{1}+\left(V_{2}-V_{1}\right) \cos \alpha\right]=\pi \\
V_{1}+\left(V_{2}-V_{1}\right) \cos (3 \alpha)=0 \\
V_{1}+\left(V_{2}-V_{1}\right) \cos (5 \alpha)=0
\end{array}\right\}
$$

Solving the set of equations one can receive: $V_{1}=0.3927, \quad V_{2}=0.9481, \quad \alpha=\frac{\pi}{4}, \quad b_{1}=1, \quad b_{3}=b_{5}=0$

The results of calculations are presented in Fig. 5. The THD ratio is $23,1 \%$.

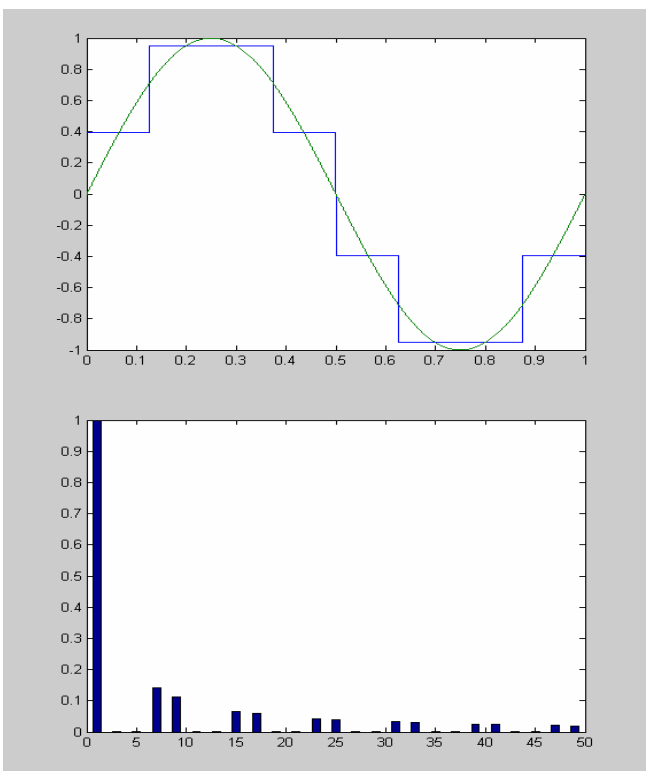

Fig. 5. The approximation of the function $f(x)=\sin (x)$ for $N=2$ $\left(\alpha_{0}=\pi / 4, \alpha_{1}=\pi / 2\right)$ with third and fifth harmonics cancelled and the spectrum analysis of the waveform

Taking as a criterion the minimal value of the THD ratio it is possible to find an optimal ratio of step parameters as a minimal value of the expression:

$$
T H D=\frac{1}{b_{1}} \sqrt{\sum_{k=1}^{k=\infty} b_{2 k+1}^{2}}
$$

Considering (11)

$$
T H D=\frac{4 V_{1}}{\pi b_{1}} \sqrt{f(\alpha, \theta)} \quad k=1,2, \ldots
$$

and the minimal value of THD is related to the minimum of the function:

$$
f(\alpha, \theta)=\sum_{k=1}^{k=\infty}\left\{\frac{1}{2 k+1}[\theta+(1-\theta) \cos [(2 k+1) \alpha]\}^{2}\right.
$$

The three dimensional picture of the analyzed function $f(\alpha, \theta)$ is presented in the Fig. 6 .
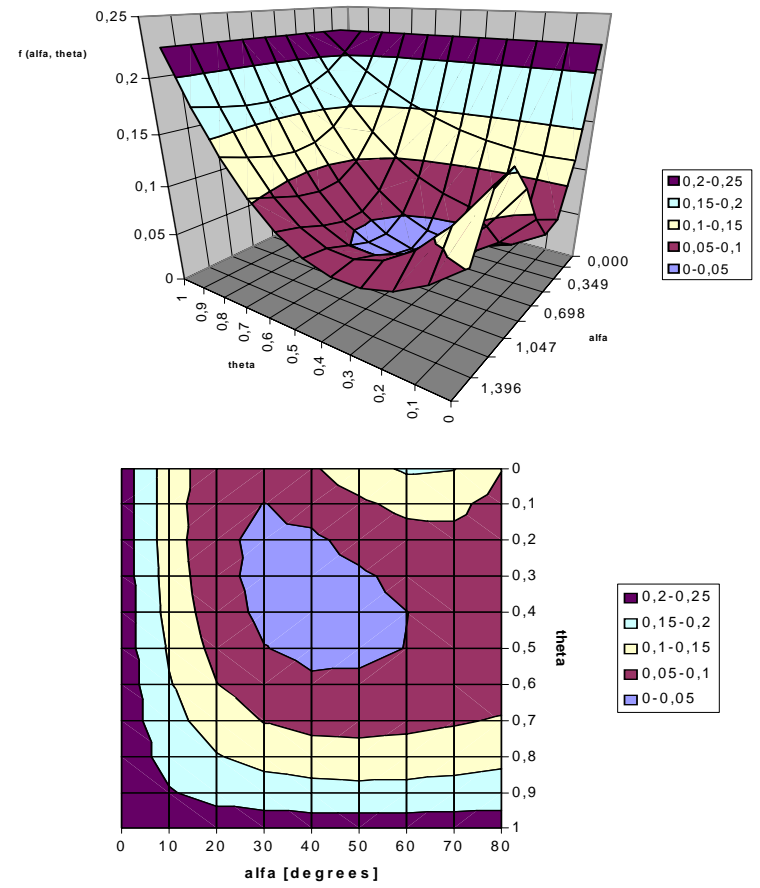

Fig. 6. Three dimensional picture of the function $f(\alpha, \theta)$, and its projection on $(\alpha, \theta)$ plane showing the optimal area

The parameters of the optimal approximation for the minimum THD value are as following: $\alpha=2 \pi / 9$ $\left(40^{\circ}\right), \theta=0.4, b_{1}=1, V_{0}=0.3655, V_{1}=0.9136$. The THD factor for this waveform is $20.40 \%$. The optimal waveform and its harmonic spectrum is presented in the Fig. 7.

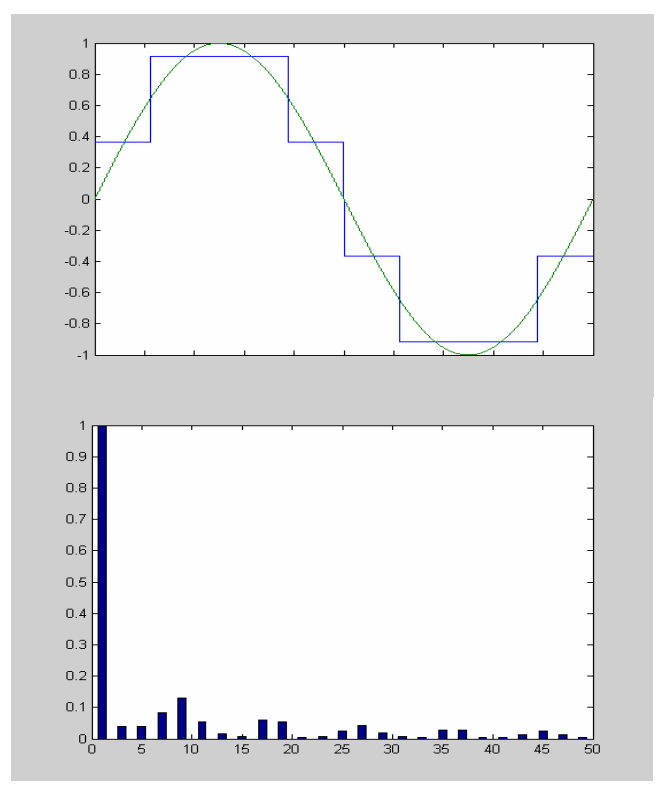


Fig. 7. The approximation of the function $f(x)=\sin (x)$ for $N=2$ and minimal THD factor with optimal parameters $\left(\alpha_{0}=2 \pi / 9\right.$,

$$
\alpha_{1}=5 \pi / 9, \mathrm{THD}=20.40 \% \text { ) }
$$

\section{APPLICATION EXAMPLES}

The proposed converter model can be used as the description of multilevel converter's output waveforms in the form of orthogonal square impulses. The output waveform is created as a sum of the consecutive impulses shifted along the $\omega t$ axis. The idea of summing impulses is presented in the Fig. 8. The presented converter consists of two one-phase bridge inverters (F1 and F2) and a summing circuit $\Sigma$.

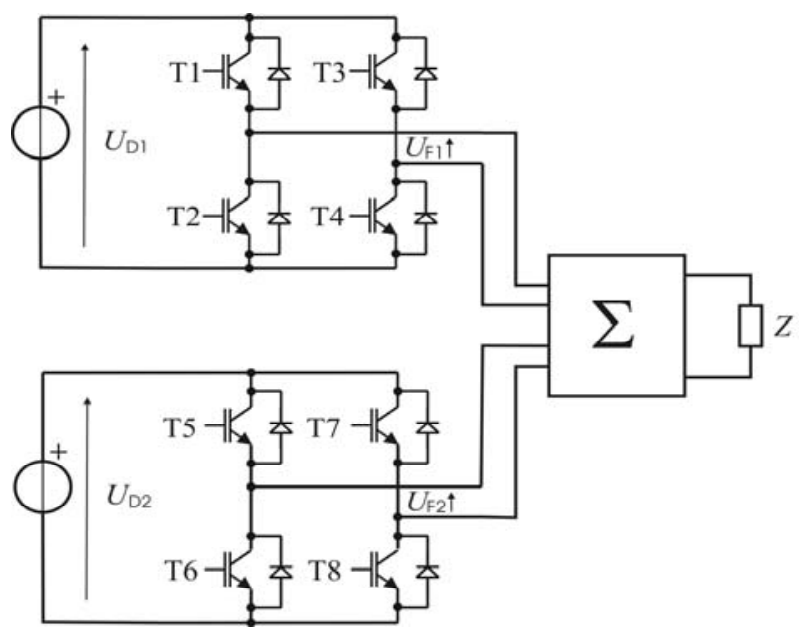

Fig. 8. The example of one-phase voltage converter generating $f_{N=6}$ waveform

The individual consecutive impulses are generated in this way that the end of the former impulse adjoins the beginning of the next one. Therefore it is possible to design direct coupled solutions (without summing circuitry), similar to cascade converters. The control principle for the individual semiconductor switches within the converter is derived directly from the presented idea of the output waveforms construction.

The three-phase three-level direct coupled voltage converter built using three one-phase four-switches inverters supplied from common voltage source is presented in the Fig. 9.

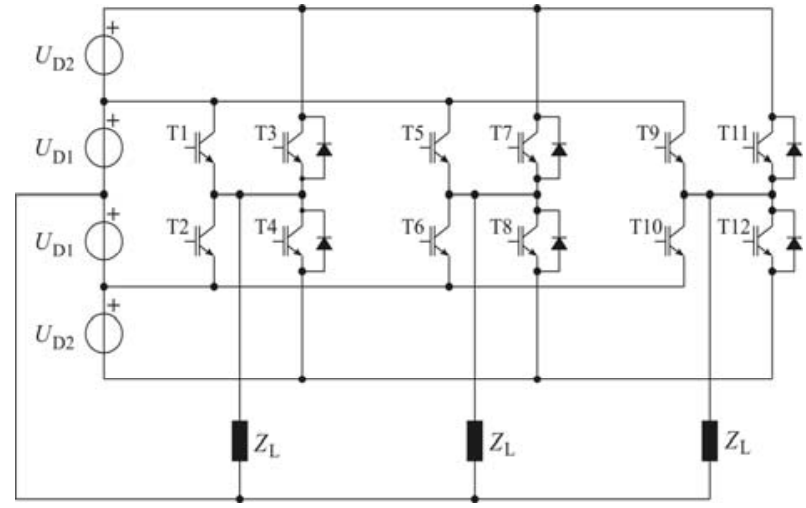

Fig. 9. Three-phase three-level voltage converter built using three one-phase four-switches inverters

\section{CONCLUSIONS}

The above described method of a sine wave approximation permits to define a mathematical model of the converter in which the synthesis of the alternate output waveform is based on the generalized Fourier series. The Fourier series contains a set of limited number of orthogonal functions representing rectangular pulses. The presented application examples show the possibilities of the practical use of the proposed method. The full description of the model and some more resulting implications are inserted in the monograph [1].

\section{REFERENCES}

[1] IWASZKIEWICZ Jan: "Mathematical Models of Power Electronics Multilevel Converters - Analysis and Applications", Proceedings of Electrotechnical Institute, vol. 227, pp. 142, Warsaw, 2006, Poland.

[2] IWASZKIEWICZ Jan., Perz Jacek: "Fourier Series and Wavelet Transform Applied to Stepped Waveforms Synthesis in Multilevel Convertors" - Proceedings of

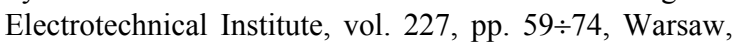
2006, Poland. 